\title{
Effects of Non-Electrostatic Intermolecular Interactions on the Phase Behavior of pH-Sensitive Polyelectrolyte Complexes
}

\author{
$\mathrm{Lu} \mathrm{Li}^{1}$, Samanvaya Srivastava2, , Siqi Meng', Jeffrey M. Ting ${ }^{1}$, Matthew V. Tirrell1,3,* \\ ${ }^{1}$ Pritzker School of Molecular Engineering, The University of Chicago, Chicago, IL 60637, USA \\ ${ }^{2}$ Chemical and Biomolecular Engineering, University of California, Los Angeles, Los Angeles, CA 90095, USA \\ ${ }^{3}$ Center for Molecular Engineering and Materials Science Division, Argonne National Laboratory, Lemont, IL 60439, USA
}

\section{KEYWORDS: Polyelectrolyte complexation, Hydrophobicity, Phase Behavior, Hydrogen Bonding}

\begin{abstract}
Polyelectrolyte complexes (PECs) offer enormous material tunability and desirable functionalities, and consequently have found broad utility in biomedical and materials industries. Poly(acrylic acid) (PAA) and poly(allylamine hydrochloride) $(\mathrm{PAH})$ are one of the most commonly used pairings to form PECs. However, various aspects of the phase behavior of PAA-PAH complexes have not been sufficiently quantified. We present a comprehensive experimental study depicting the binodal phase boundaries for the PAA-PAH complexes prepared in acidic, neutral and basic conditions using thermogravimetric analysis, turbidimetry and optical microscopy. In neutral and basic conditions, phase behaviors of the complexes were largely similar to each other and followed general expectations of PEC phase behavior, except for unusually high salt resistance with stable complexes observed up to $4 \mathrm{M} \mathrm{NaCl}$ concentrations. In acidic conditions, a remarkably different phase behavior of the PAA-PAH complexes was observed. The polymer content in the complex phase increased initially followed by an expected decrease as salt was added to the complexes. This behavior may result from a combination of associative phase separation of PAA and PAH chains, influenced by electrostatic interactions, and segregative phase separation which can be ascribed to the influence of a combination of the hydrophobic interactions of the aliphatic polymer backbone and the interpolymer hydrogen bonding of unionized acrylic monomer units. Our systematic investigations detailing these discrepancies in the PAA-PAH phase behavior are expected to clarify the inconsistencies among the reports in the literature and inform the materials design strategies for practical use of the PAA-PAH complexes and multilayer assemblies.
\end{abstract}

Mixing of oppositely charged polyelectrolyte solutions in aqueous solutions can result associative phase separation of the polyelectrolytes leading to polyelectrolyte complexes (PECs). ${ }^{1-5}$ With increasing awareness of striking parallels in biology, e.g. the resemblance of PEC to membraneless organelles emerging from spontaneous liquid-liquid phase separation,, 67 and material science as exceptionally tunable self-assembled structures, ${ }^{8,9}$ PEC-based materials with tunable macroscopic properties and functionalities have become increasingly popular across multiple disciplines. For instance, they have been employed as prototypes in reversible morphological phase transitions, ${ }^{10,11}$ fabricated into hollow microcapsules and intertwined membranes by injection suspension or electron spinning, $8,12,13$ and formulated into therapeutic delivery micelles ${ }^{14-16}$ as well as biocompatible hydrogels. ${ }^{9}$

Several experimental ${ }^{13,17-23}$ and theoretical studies ${ }^{24-31}$ in recent years have investigated the thermodynamics ${ }^{23,32,33}$ and kinetics $^{34,35}$ of polyelectrolyte complexation, focusing on the influence of electrostatic interactions and the concomitant entropic gains from the release of counterions upon complexation. Only a handful of theoretical and simulation works have investigated the influence of polymer-solvent interactions on the phase behavior of complexes. ${ }^{36,37}$ Nakajima et al. included the Flory interaction parameter $\chi$ to account for interaction between water and polyelectrolyte salt in the classical Voorn-Overbeek (V-O) theory ${ }^{3}$ and deduced the limiting conditions of charge density and chain length for complexation $^{38,39}$. Larson and coworkers extended the $\mathrm{V}-\mathrm{O}$ theory ${ }^{3}$ by incorporating the $\chi$ parameter between protonated polyacrylic acid and solvent molecules to explain the usually high salt resistances of PECs measured in acidic environments. ${ }^{36,40,41} \mathrm{~A}$ recent theoretical study by Rumyantsev et al. outlined a salt concentration-solvent quality diagram of PECs based on scaling laws. ${ }^{37}$ Concomitantly, experimental investigations have begun to investigate the influence of parameters like unique chemical attributes of the individual polymers and solvent effects on complexation. . $6,37,42$ Sadman et al. have demonstrated mechanical tunability for poly(styrene sulfonate) and poly(4-vinyl pyridine) complexes by varying the hydrophobicity of the quaternized poly(4vinylpyridine) through alkyne substitutions, ${ }^{42}$ and Spruijt et al. included the $\chi$ parameter in calculations based on $\mathrm{V}-\mathrm{O}$ theory to describe experimentally determined polymer contents in PECs. ${ }^{43}$

Despite the progress in the description of the influence of solvent interactions on complexation, detailed datasets describing the phase behavior quantitatively to illustrate the effect of hydrophobicity and other prevailing physical noncovalent interactions on polyelectrolyte complexation are still 


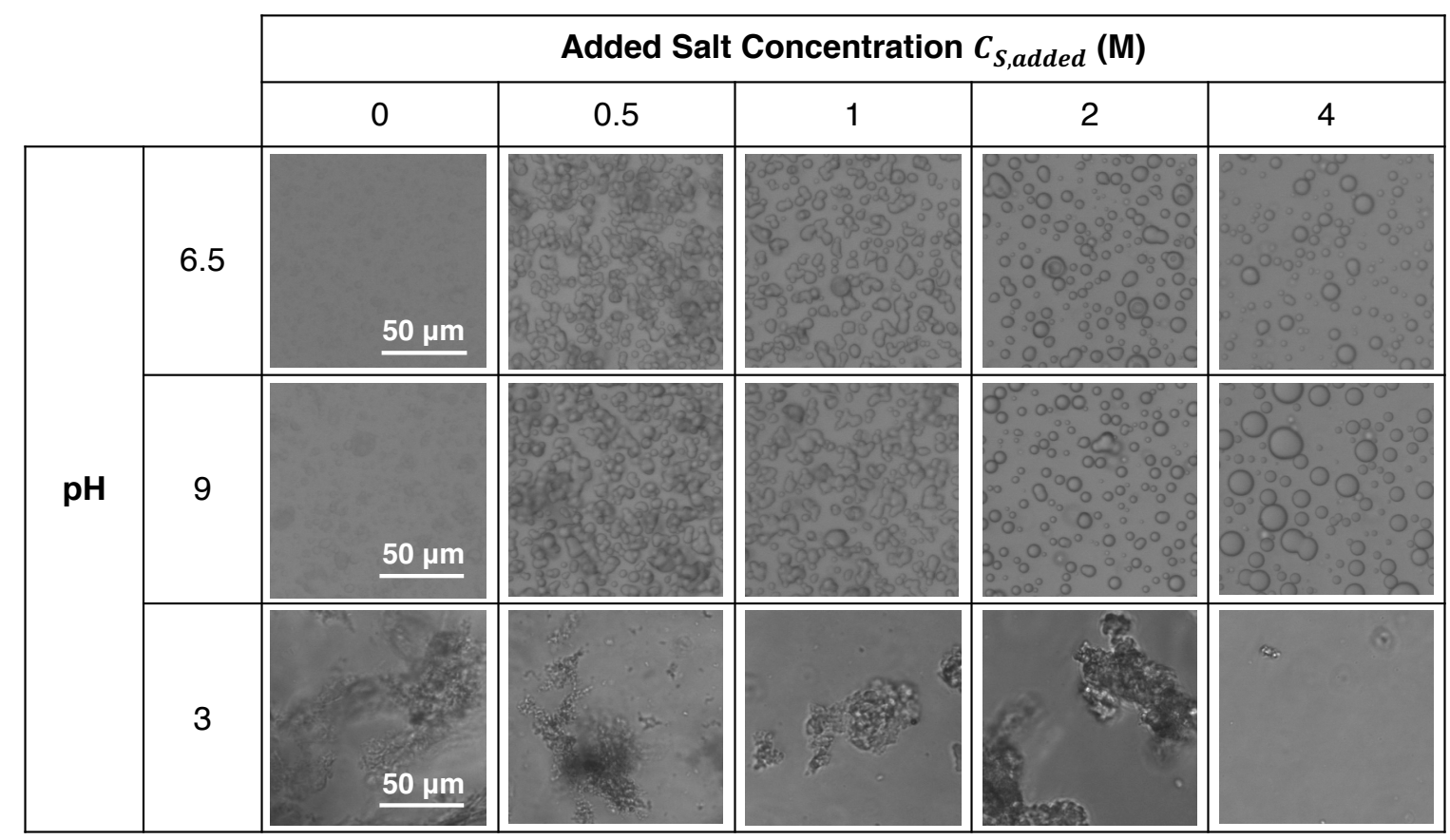

Figure 1. Representative micrographs of (rows 1, 2) PAANa-PAH and (row 3) PAA-PAH complexes prepared at $C_{P, 0}=1 \%$ wt $/ v, C_{S, a d d e d}$ $=0,0.5,1,2$, and $4 \mathrm{M} \mathrm{NaCl}$ and $\mathrm{pH}=6.5$ (row 1), 9 (row 2) and 3 (row 3). At $\mathrm{pH}=6.5$ and 9, the morphology of the complexes changed from flaky precipitates to irregularly shaped droplets to spherical droplets with increasing $C_{S, \text { added }}$. At $\mathrm{pH}=3$, the complexes remained as flaky precipitates for all $C_{S, \text { added }}$ investigated. The scale bar is the same for all images.

elusive. These more realistic aspects of complexation are essential to advance our current understanding of selecting polyelectrolyte pairs for PEC materials and to harness such materials into products and end-use technologies.

Here, we provide explicit quantitative descriptions of the binodal phase behavior of polyelectrolyte complexes comprising polyelectrolyte pairs with polarizable ionic groups on aliphatic backbones, resulting in accentuated hydrophobicity and hydrogen bonding interactions in conjunction with electrostatic interactions. Sodium salt of poly(acrylic acid) (PAANa) (and its acid form, polyacrylic acid, PAA) with poly(allylamine hydrochloride) (PAH) were the polyelectrolytes of choice for this study due to their commercial relevance and widespread application in both academia and industry. ${ }^{8,44-48}$ Despite their extensive use, the interplay between electrostatic and other non-covalent interactions on complexation between these two polymers remains unclear, resulting in inconsistencies among reports on their phase behaviors, complex morphologies and stability against salt in varying environments. On one hand, Chollakup et al. investigated the salt resistance and amount of PAA-PAH complexes and reported maximum complexation along with maximum salt resistance ( 1-1.5 M) of complexes around $\mathrm{pH}$ $=7,44,49$ and Fu et al. ranked PAA as the weakest polyanion to complex with and smallest ion-pairing strength among three other commonly used polyanions, and consequently PAA related complexes are expected to easily dissolve in presence of external salt. ${ }^{50}$ On the other hand, several studies have reported that PAA-based complexes prepared in acidic conditions exhibit high salt resistances.21,36,51 For example, Larson and coworkers pointed out despite the pKa of PAA being $\sim 5-5.5$ and of PAH being $\sim 8.5-9.3,{ }^{52}$ the salt concentrations required to induce morphological transitions from precipitate to coacervate and from coacervate to solution were markedly asymmetric around $\mathrm{pH} 7$ in PAA-PAH complexes, and a critical $\mathrm{KCl}$ concentrations $>3 \mathrm{M}$ were required to completely dissolve the complexes; both these observations could not be explained solely by electrostatic interactions. ${ }^{36}$ Lappan et al. reported significantly slower dynamics of PAA chains in complexes prepared in acidic conditions, ${ }^{53}$ suggesting stronger association of the polymer network in low $\mathrm{pH}$ conditions. Thus, a comprehensive study of the phase behavior of PAA-PAH at various $\mathrm{pH}$ conditions is required to address the inconsistencies in PAA-PAH phase behavior. We address these inconsistencies by highlighting the role of hydrophobic interactions of the polyelectrolyte backbones as well as hydrogen bonding on the phase behavior and salt resistance of PAA-PAH complexes. The contributions from these non-electrostatic interactions are shown to become especially prominent in high salt concentrations or in acidic conditions, wherein the electrostatic interactions are screened or weakened, respectively.

Mixing of PAANa and PAH (degree of polymerizations 158 and 160, respectively, Supplementary Table 1) at equivalent monomer concentrations (to ensure charge matching), controlled total polymer concentration and added salt $(\mathrm{NaCl})$ concentration, $C_{P, 0}$ and $C_{S, \text { added, }}$ respectively, led to complexation instantly. The mixture became turbid with flaky 
white precipitates ${ }^{46,49}$ suspended in the solution (Supplementary Figure 1). The first row in Figure 1 shows representative micrographs of the sedimented complexes with increasing concentration of added salt $C_{S \text {,added }}$ in PAANa-PAH complexes with total polymer concentration $C_{P, 0}=5 \% w t / v$ at $\mathrm{pH}=6.5$. Flaky PEC precipitates in the absence of added salt $\left(C_{S, \text { added }}=0 \mathrm{M}\right)$ transitioned to gel-like irregularly shaped droplets to liquid-like spherical PEC droplets with increasing $C_{S, \text { added }}$, indicative of increasing water content in the complexes. Interestingly, complexation persisted up till $C_{S \text {,added }}=4 \mathrm{M}$, which is particularly high when contrasted with previous reports in the literature. ${ }^{44,49}$ Moreover, addition of $\mathrm{KBr}$, reported frequently as an effective salt in disrupting complexation, ${ }^{42,50}$ also did not dissolve the complexes up to $4 \mathrm{M}$ added salt concentration (Supplementary Figure 2). ${ }^{42,44,49}$

The salt concentration required to eliminate phase separation, referred to as the salt resistance $\left(C_{S R}\right)$ of the complexes, is often obtained using turbidimetry. ${ }^{11,17,49,54-59}$ Figure 2a shows a typical evolution of turbidity with increasing $C_{S, \text { added }}$ in PAANa-PAH complexes with total polymer concentration $C_{P, 0}=5 \% w t / v$ at $\mathrm{pH}=6.5$. Turbidity of the solution decreased with increasing $C_{S \text {,added }}$ and was ascribed to decreasing volume of complexes arising from increasing screening of electrostatic interactions among the oppositely charged chains. Solution turbidity diminished and plateaued at $C_{S R}=2.0 \mathrm{M}$. Similar evolution of turbidity with $C_{S, \text { added }}$ for complexes with varying $C_{P, 0}$ were observed (Supplementary Figure 3 ). The vanishing of turbidity, however, could not be correlated with complete dissolution of the complexes. At high $C_{S, \text { added }}$ values $(\geq 2 \mathrm{M})$, the complexes sedimented to the bottom of the solutions, leading to clear supernatant phases. These precipitates were evident from a microscopic inspection of the solutions and are shown in Figure 1 and the inset of Figure 2a. These observations may explain the inconsistencies in the previously reported results from different studies which relied on turbidimetric analysis to identify phase separation. ${ }^{36,44,49,50,60}$ Since Chollakup et al. ${ }^{44,49}$ used small total polymer concentrations in preparing the complexes $\left(C_{P, 0}=0.05 \% w t / v\right)$, the complex phases may have been too small to be identified and produce turbidity in the solution, especially at high salt concentrations. Therefore, the reported salt resistance values, obtained from turbidity analysis, may have been non-representative. Additionally, the large and dense droplets and semi-solid precipitates that form upon complexation of PAA and PAH accumulate and sediment to the bottom of the container quickly, resulting in low turbidity, especially if samples are allowed to equilibrate. Therefore, as a guideline, combining turbidimetry with other visual characterization techniques like optical microscopy is recommended to unambiguously identify phase transitions in PEC solutions. Furthermore, immediate measurements of turbidity after sample preparation are recommended. For microscopic imaging, especially at low total polymer
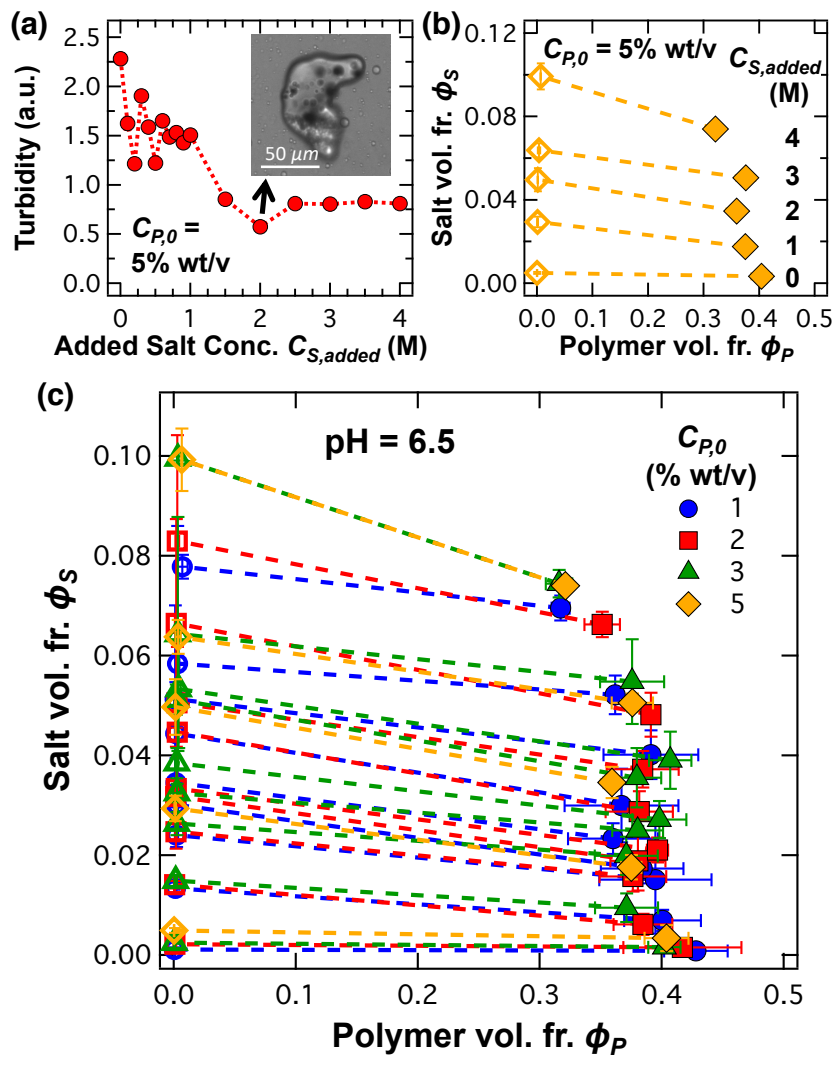

Figure 2. (a) Turbidity, and (b) composition map depicting the total polymer and total salt content in the complex and supernatant phases, of polymer-charge-matched mixtures comprising $\mathrm{PAANa}_{158}$ (sodium salt), $\mathrm{PAH}_{160}$ and $\mathrm{NaCl}$ prepared in neutral condition $(\mathrm{pH}=$ 6.5) at a total polymer concentration $C_{P, 0}=5 \% \mathrm{wt} / v$ and added salt concentrations $C_{S \text {, added }}$ varying from $0-4 \mathrm{M}$. In (b), the filled (complex phase) and open (supernatant phase) symbols are connected by dotted coexistence lines. (c) Overlaid binodal phase boundaries of mixtures described in (b) prepared at total polymer concentration $C_{P, 0}=1 \%, 2 \%, 3 \%$, and $5 \% \mathrm{wt} / v$ and added salt concentrations varying from $0-4 \mathrm{M}$. Data in (b) and (c) were obtained from thermogravimetric analysis of the complex and the supernatant phases. Error bars in (b) and (c) denote the standard deviations in each measurement.

concentrations, centrifugation of the complexes is recommended to facilitate imaging of the complexes.

A quantification of PEC phase behavior was pursued through compositional analysis (water, polymer, and salt contents) of the complex and the supernatant phases using thermogravimetric analysis (TGA) protocol developed in our previous report ${ }^{61}$ (see Supporting Information for experimental protocols). Figure $\mathbf{2} \mathbf{b}$ shows the binodal phase boundaries for PAANa-PAH complexes at $C_{P, 0}=5 \% w t / v$ in neutral conditions $(\mathrm{pH}=6.5)$ mapped on the polymer concentration $\left(\phi_{P}\right)$ - salt concentration $\left(\phi_{S}\right)$ space. Pairs of points, connected by dashed lines, correspond to the compositions of the two phases resulting from bulk phase separation of complex and supernatant phases, represented by filled and unfilled symbols, respectively. The complex 
phase was found to have slightly smaller counterion concentration than the supernatant phase, as depicted by the negatively slopes of the lines connecting the compositions of the two phases. This observation is contrary to $\mathrm{V}-\mathrm{O}$ theory but in agreement with our previous report ${ }^{61}$ and other recent reports. ${ }^{13,29,61,62}$

Trends in binodal phase behaviors at neutral conditions were largely conserved at other $C_{P, 0}$ values $(=1,2$ and $3 \% w t / v$, Supplementary Figure 4). The polymer content of the complex phase $\phi_{P}$ generally decreased with increasing $C_{S \text {,added, attributed to the screening of electrostatic }}$ interactions by additional of salt, in accordance with the Voorn-Overbeek (V-O) theory ${ }^{3}$ and previous studies. ${ }^{20,26,43}$ Figure 2c shows the superposition of binodal phase boundaries for PAANa-PAH complexes at $\mathrm{pH}=6.5$ for $C_{P, 0}$ ranging from $1 \%$ to $5 \% w t / v$, and shows a good overlap of the data from different set of experiments. In most cases, the salt ions were found to partition preferentially into the supernatant phases. This salt partitioning behavior can be surmised to be consistent irrespective of the hydrophobic or hydrophilic ${ }^{3,5,61}$ nature of the backbone. It must be noted that preferential partitioning of salt into the complex phase may yet be imposed by molecular engineering of the polymer and counterion identities (different chemical structures, valency, and physical features). ${ }^{62,63}$

Upon closer inspection, however, unique trends emerged in the binodal phase boundaries. The merging of the complex branch with the supernatant branch, an indication of the transition from the two-phase region into one homogenous phase, was not observed even up to $4.0 \mathrm{M}$ of added $\mathrm{NaCl}$ (Figure 2b), consistent with observation of complexes in microscopy experiments (Figure 1, first row). Further, $\phi_{P}$ initially decreased with increasing $C_{S, \text { added }}$ in the range of $0<$ $C_{S \text {,added }}<0.5 \mathrm{M}$, then plateaued for $0.5<C_{S \text {, added }}<1.5 \mathrm{M}$ before decreasing again for $C_{S, \text { added }}>1.5 \mathrm{M}$. These behaviors are distinct from previous reports ${ }^{13,21,64}$ on PEC phase behavior where $\phi_{P}$ monotonically and continually decreased with increasing $C_{S, \text { added, }}$ and can be tentatively ascribed to the increasing influence of non-electrostatic intermolecular interactions on the complex phase behavior.

Morphology of PAANa-PAH complexes in basic conditions $(\mathrm{pH}=9)$ exhibited an evolution with $C_{S, \text { added }}$ similar to the complexes in neutral conditions (second row in Figure 1). The binodal phase boundaries of PAANa-PAH complexes in basic conditions, obtained by superposing the composition data from PECs prepared with varying $C_{P, 0}$, are summarized in Figure 3. The composition of the two phases and the shape of the phase envelope was similar to the map shown in Figure 2c (individual data sets for different $C_{P, 0}$ are compiled in Supplementary Figure 5). Further, similar to complexes in neutral conditions, $\phi_{P}$ plateaued for PECs with $0.5 \mathrm{M}<$ $C_{S, \text { added }}<1.5 \mathrm{M}$ (Supplementary Figure 5).

Since both PAANa and PAH are weak polyelectrolytes, their ionization is strongly affected by the $\mathrm{pH}$ of the

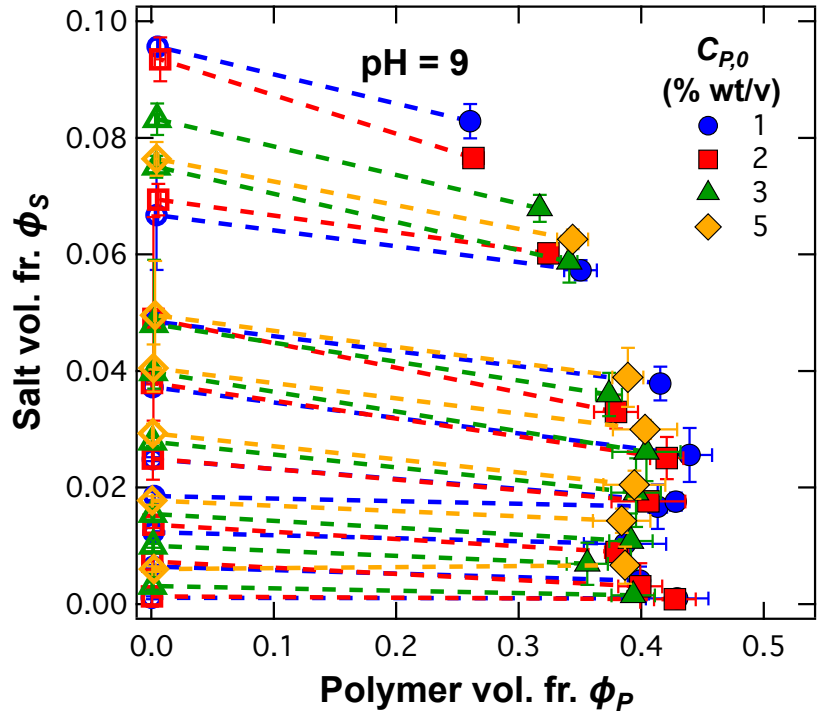

Figure 3. Overlaid binodal phase boundaries depicting the total polymer and total salt content in the complex and supernatant phases, obtained from thermogravimetric analysis, of polymercharge-matched mixtures comprising $\mathrm{PAANa}_{158}$ (sodium salt), $\mathrm{PAH}_{160}$ and $\mathrm{NaCl}$ prepared in basic condition $(\mathrm{pH}=9)$ at total polymer concentrations $C_{P, 0}=1 \%, 2 \%, 3 \%$, and $5 \% \mathrm{wt} / v$ and added salt concentrations varying from $0-4 \mathrm{M}$. The filled (complex phase) and open (supernatant phase) symbols are connected by dotted coexistence lines. Error bars denote the standard deviations in each measurement.

environment. In basic conditions, PAH ionized partially $(24 \%$, Supplementary Figure 6), in effect reducing the charge density of the polyelectrolyte and propensity for complexation. ${ }^{65}$ The similarity of the overall phase boundaries obtained in neutral and basic conditions, however, points towards the analogous role of electrostatic and other intermolecular interactions in dictating the PEC phase behavior is these conditions, with the partial ionization of PAH only contributing to diminished electrostatic correlation and increased water contents in the complexes. Further, we hypothesize that cooperativity of electrostatic interactions between cationic and anionic chains can result in promoting the ionization of $\mathrm{PAH}$ chains. ${ }^{66,67} \mathrm{We}$ expect these observations to inspire theoretical and simulation investigations, that in turn will provide further insights into the phenomena.

Morphology of PECs comprising the acidic form of PAA (degree of polymerization 138, Supplementary Table 1) with PAH prepared in acidic conditions $(\mathrm{pH}=3)$, however, were remarkably different compared with those obtained in basic and neutral conditions (third row in Figure 1). This adjustment of the polyanion was required because PAANa was found to precipitate in $\mathrm{pH}=3$ solutions. In contrast to the continuous evolution of complex morphology with increasing $C_{S \text {,added }}$ in neutral and basic conditions, the complexes prepared in acidic conditions were found to retain their flaky 
precipitate morphology even at the highest $C_{S \text {,added }}$ investigated (third row in Figure 1).

Binodal phase boundaries of complexes prepared in acidic conditions were also remarkably different compared with those obtained in basic and neutral conditions, particularly in the low $\phi_{S}$ region. Figure 4a exemplified the distinctive phase diagram for $C_{P, 0}=3 \% w_{t} / v$; Figure $4 \mathbf{b}$ presents the superimposed phase diagrams at $C_{P, 0}=1,2,3$, and $5 \% w t / v$ with $C_{S, \text { added }}$ ranging from 0 to $4 \mathrm{M}$ (individual data sets for different $C_{P, 0}$ are compiled in Supplementary Figure 7). Specifically, the complex branch extended right towards higher $\phi_{P}$ with increasing $C_{S \text {,added }}$ until $C_{S \text {,added }}<1 \mathrm{M}$, which indicated enriching of polyelectrolyte content in the complex phase with addition of salt, opposite of the trends from the other two conditions. For $C_{S, \text { added }}>1 \mathrm{M}$, the trends of decreasing $\phi_{P}$ with increasing $C_{S \text {, added }}$ were recovered. The composition data obtained from different $C_{P, 0}$ and $C_{S, \text { added }}$ overlapped reasonably well (Figure $4 \mathbf{b}$ ), which indicated that this unusual trend of increasing polymer content in the complex phase upon increasing salt concentration in the solution was consistent regardless of starting point on the composition map. However, the phase separation was found to be $C_{P, 0}$ dependent, as evident from the significant differences in the complex compositions at high $C_{S \text {,added }}$ and from the intersecting coexistence lines (denoted by dashed lines in Figure $\mathbf{4 b}$ ). It must be noted that $C_{P, 0}$-independent phase behavior results in non-intersecting phase coexistence lines, as shown in Figures $2 \mathrm{c}$ and 3 . Additionally, the complexes were found to be asymmetric in concentration of PAA and PAH in acidic conditions owing to partial ionization of PAA chains in the acidic conditions. ${ }^{1} \mathrm{H}$ NMR spectra of the complexes prepared at $C_{P, 0}=3 \% \mathrm{wt} / v$ and $C_{S, \text { added }}=2.0 \mathrm{M}$ indeed showed that the molar ratio of PAA to PAH was 0.67 in complexes prepared in acidic conditions, as compared to 0.43 and 0.40 in their counterparts prepared in neutral and basic conditions, respectively (Supplementary Figure 8, Supplementary Table 3), in good agreement with previous literatures. 8,36 A combination of associative phase separation of PAA and PAH as well as segregative phase separation of PAA could thus be argued to result in the formation of complexes in acidic conditions.

These unique trends in the composition maps appears similar to the salting in and salting out behavior of polyelectrolytes (with strong electrostatic interactions) that can occur upon increasing concentration of monovalent and multivalent salt, as has been suggested by Zhang et al. ${ }^{30}$ In polyelectrolyte solutions, this behavior was proposed to arise from an interplay between increasing translational entropy of salt ions and excluded volume interactions promoting homogenization of the solution competing with electrostatic correlations favoring phase separation. In PEC systems, salting-out is typically not observed owing to small translational entropy of the oppositely charged polyelectrolyte chains dominated by the strong electrostatic correlations between them. With increasing salt
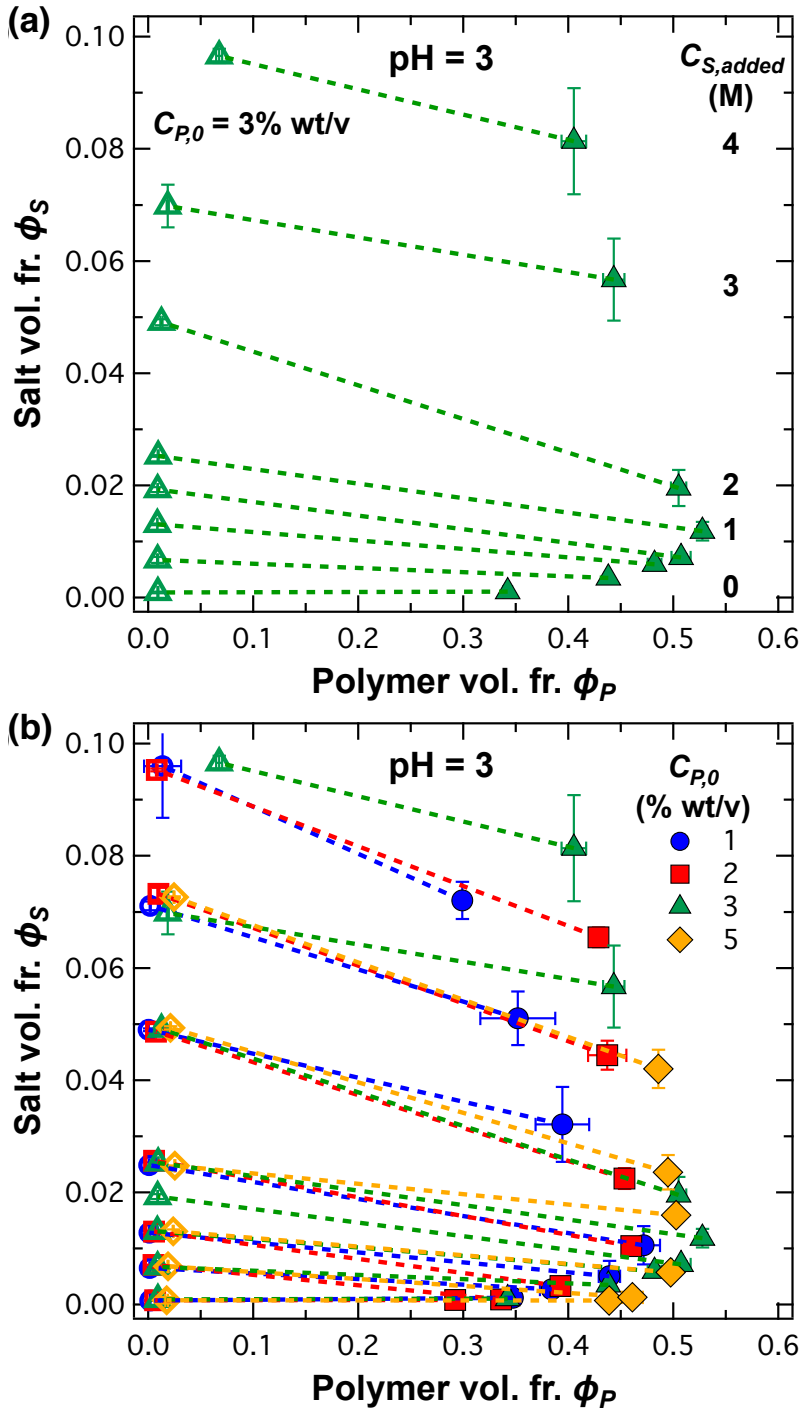

Figure 4. (a) A composition map depicting the total polymer and total salt content in the complex and supernatant phases, obtained from thermogravimetric analysis, of polymer-charge-matched mixtures comprising $\mathrm{PAA}_{138}, \mathrm{PAH}_{160}$ and $\mathrm{NaCl}$ prepared in acidic condition $(\mathrm{pH}=3)$ at a total polymer concentration $C_{P, 0}=3 \% \mathrm{wt} / \mathrm{v}$ and added salt concentrations varying from $0-4 \mathrm{M}$. The filled (complex phase) and open (supernatant phase) symbols are connected by dotted coexistence lines. (b) Overlaid binodal phase boundaries of mixtures described in (b) prepared at total polymer concentration $C_{P, 0}=1 \%, 2 \%, 3 \%$, and $5 \% \mathrm{wt} / v$ and added salt concentrations varying from $0-4 \mathrm{M}$. Error bars in (a) and (b) denote the standard deviations in each measurement.

concentrations, however, a salting-in phenomenon is consistently observed. ${ }^{5}$ In PAA-PAH complexes in acidic conditions, however, PAA is expected to partially ionize (3\%, Supplementary Figure 6), resulting in weak electrostatic correlations between the PAA and PAH chains. Electrostatic correlations between the oppositely charged chains, mediated by monovalent counterions ions, are expected to increase initially with increasing $C_{S, \text { added }}$, leading to increasing $\phi_{P}$ in the PECs. At higher $C_{S, \text { added }}$, counterion entropy and excluded 
volume interactions eventually dominate the phase behavior, resulting in decreasing $\phi_{P}$ in the PECs. We note that salting in and salting out phenomena has been described in the literature for four component systems (polyelectrolyte, salt ions and water) while the system considered here is a five component system comprising two polyelectrolytes, limiting the conclusion that could be drawn from the theoretical descriptions of the former for the latter systems.

In addition to lower ionization of the PAA (3\%, Supplementary Figure 6) chains leading to weaker electrostatic correlations, hydrophobic interactions of the hydrocarbon backbone of the partially-ionized PAA chains with water and the hydrogen bonding of acrylic acid moieties are also expected to influence the PEC phase behavior. ${ }^{68,69}$ The unionized functional groups in PAA can serve as hydrogen bond donors and/or acceptors; Buscall and Corner proposed that hydrogen bonding can be facilitated under acidic conditions for PAA. ${ }^{69}$ Upon addition of salt, both hydration and hydrogen bonding are disrupted, owing to screening of electrostatic interactions by the salt ions and the reduced availability of water molecules to form hydrogen bonds. Phase separation of the PAA from water may occur in such a case upon formation of intra- and inter-chain hydrogen bonds among the ionized acrylic acid moieties, accompanied by the unfavorable interactions of the unionized aliphatic backbone with water.

The influence of salt on solubility of individual polyelectrolytes, PAA and PAH, in water was investigated using turbidimetric analysis and optical microscopy. While $\mathrm{PAH}$ solutions remained stable across the $\mathrm{pH}$ range investigated, PAA was found to phase separate even at salt concentrations as low as $0.15 \mathrm{M}$. Figure 5a shows the turbidity of 5\% $w t / v$ PAA and 5\% $w t / v$ PAH solutions as a function of salt concentration. While the PAH solutions remained clear upon addition of salt, the absorbance of the PAA solution steadily increased beginning at $0.15 \mathrm{M} \mathrm{NaCl}$ concentrations, indicating phase separation of PAA. Aggregates could also be identified upon visual and microscopic inspection. At salt concentration $>0.5 \mathrm{M}$, PAA precipitates began to agglomerate and sediment at the bottom of solution, leading to lower turbidity. Eventually turbidity returned to baseline following complete sedimentation of PAA agglomerates. Inset in Figure 5 a shows a representative microscopic image of 5\% $w t / v$ PAA solution at $\mathrm{pH}=3$ with $2.0 \mathrm{M} \mathrm{NaCl}$ with considerable precipitation. Increasing $\phi_{P}$ in the complex phase with increasing $\phi_{S \text {,added }}$ for $\phi_{S, \text { added }}<1 \mathrm{M}$ and the asymmetry in the polymer content in the complexes can thus be attributed partially to this precipitation of PAA in saline solutions. Again, we note that the behavior of PAA chain in salt solutions may be different from the PAA-PAH solutions owing to strong electrostatic interactions, cooperative polyelectrolyte ionization and diminished role of counterion entropy in dictating stability of the complexed polyelectrolyte chains in the PEC systems.

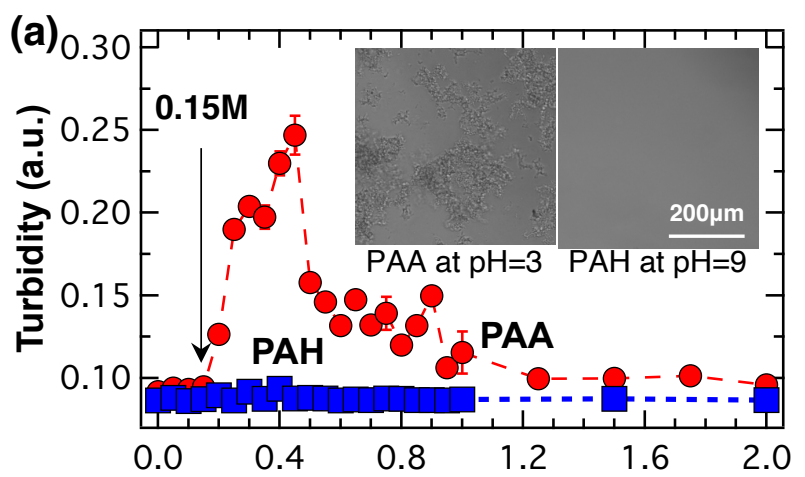

(b) Salt Concentration (M)
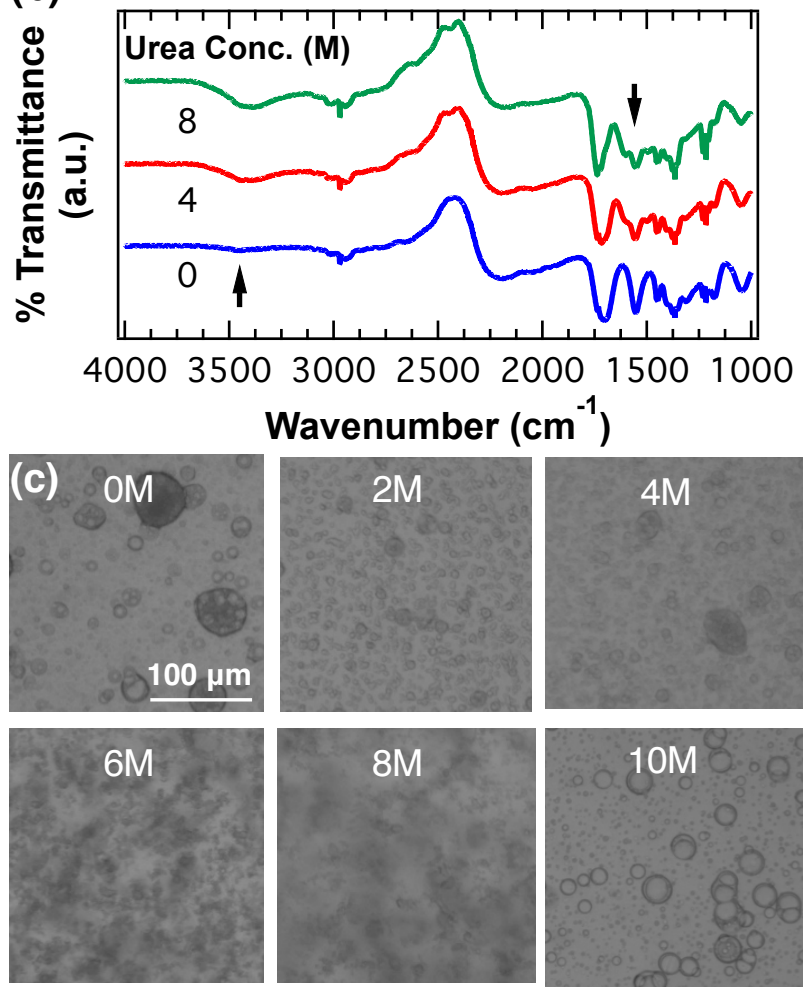

$12 \mathrm{M}$
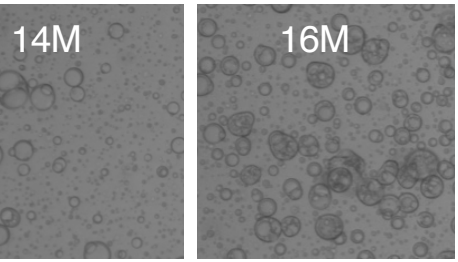

Figure 5. (a) Turbidity of aqueous PAA and PAH solutions (1\% $w t / v)$ with increasing salt concentration. Increasing turbidity upon addition of salt $>0.15 \mathrm{M}$ corresponded to PAA precipitation. Subsequent decrease in turbidity at higher salt concentrations resulted from sedimentation of the PAA precipitates. Insets depict representative micrographs of $5 \% w t / v$ PAA $(\mathrm{pH}=3)$ and $\mathrm{PAH}(\mathrm{pH}$ =9) solutions with $2 \mathrm{M} \mathrm{NaCl}$. The scale bars were the same for both images. (b) FTIR spectra of PAA-PAH PECs prepared in $\mathrm{pH}=3$ solutions with 0,4 , and $8 \mathrm{M}$ of urea. Arrows at $\sim 3450 \mathrm{~cm}^{-1}$ and $\sim 1560 \mathrm{~cm}^{-1}$ indicate free $\mathrm{O}-\mathrm{H}$ stretching vibrations and hydrogen bonding of the deprotonated carboxylic acids, respectively. (c) Micrographs of the complexes with $C_{P, 0}=1 \% \mathrm{wt} / v, C_{S, \text { added }}=2 \mathrm{M}$ and $\mathrm{pH}=3$ and urea concentration ranging from 0 to $16 \mathrm{M}$. The scale bars are the same for all images. 
At the same time, the effect of hydrogen bonding on PEC phase behavior in acidic conditions was examined by preparing PECs in deuterium oxide $\left(\mathrm{D}_{2} \mathrm{O}\right)$ with increasing concentrations of urea to progressively inhibit hydrogen bonding in the system. Fourier transform infrared (FTIR) spectra of PAA-PAH complexes with 0,4 , and $8 \mathrm{M}$ urea, shown in Figure $\mathbf{5 b}$, show clear evidence of weakened intermolecular or intramolecular hydrogen bonding between PAA chains. Specifically, band sharpening at $\sim 3450 \mathrm{~cm}^{-1}$ correspond to increasing $\mathrm{O}-\mathrm{H}$ stretching as urea concentration increased and broadening of a sharp single peak at $\sim 1560 \mathrm{~cm}^{-}$ ${ }^{1}$ into a bimodal peak correspond to urea forming hydrogen bonds with deprotonated $-\mathrm{COOH}$ moieties. These results indicate disruption of hydrogen bonding among PAA chains upon addition of urea.

The morphology of the PECs also varied distinctively with increasing urea concentrations. Figure $5 \mathrm{c}$ displays a representative collection of micrographs for the PAA-PAH complexes prepared at $C_{P, 0}=1 \% w t / v, C_{S, \text { added }}=2.0 \mathrm{M}$ and $\mathrm{pH}$ $=3$ with increasing urea concentration from 0 to $16 \mathrm{M}$ (the maximum achievable concentration of urea at $20{ }^{\circ} \mathrm{C}$ ). PEC morphology changed from viscous gel to flaky precipitates and finally to spherical agglomerates. Correspondingly, the weight fraction of water in the PECs, measured by thermogravimetric analysis, increased from $41.39 \%$ to $57.95 \%$ (Supplementary Table 2). Thus, it could be surmised that urea influenced the intermolecular interactions between the oppositely charged chains and PEC phase behavior in a nontrivial manner by weakening the hydrogen-bonded acrylic networks. At the same time, the close association of primary amine group of PAH with carboxyl group on PAA also contributes to the high salt resistance as indicated by Fu et al. ${ }^{50}$ Yet, since the complexes persisted and exhibited reasonably high salt tolerance under exceptionally high urea contents wherein hydrogen bonding is diminished, it could be argued that while hydrogen bonding promotes precipitation of PAA upon addition of salt, it could not be the sole contributor towards the unusual high salt tolerance of PAA-PAH PECs.

Larson and coworkers reported similar experimental observations of unusually high, $\mathrm{pH}$-asymmetric salt resistance of the PECs comprising PAA and poly $(\mathrm{N}, \mathrm{N}$ dimethylaminoethyl methacrylate) (PDMAEMA). ${ }^{36}$ Atomistic simulations showed unionized and charged acrylic acid moieties forming 2.5 and 6 hydrogen bonds with water, highlighting the hydrophobic nature of the unionized acrylic acid. ${ }^{36}$ At the same time, both neutral and charged PDMAEMA monomers formed 2 hydrogen bonds with water, emphasizing the role of PAA in influencing the phase behavior of PAA-PDMAEMA system. We expect similar factors to influence the phase behavior and salt resistance of PAA-PAH PECs.

The effect of backbone hydrophobicity, hydrogen bonding and the influence of salt on polyelectrolyte-solvent interactions can be described by an effective interaction
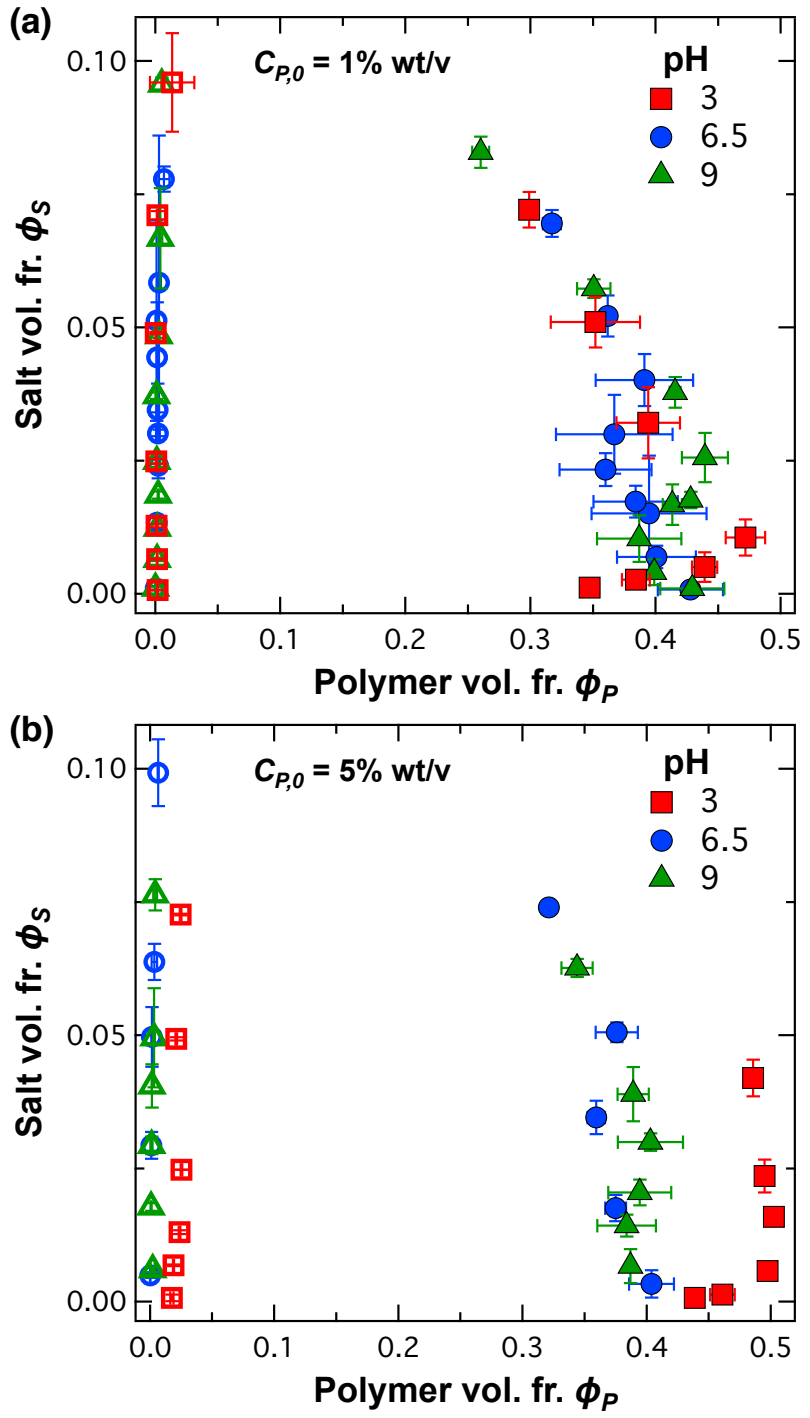

Figure 6. A comparison of the binodal phase boundaries for PAA(PAANa)-PAH complexes prepared in three different $\mathrm{pH}$ conditions with $C_{P, 0}=$ (a) $1 \% \mathrm{wt} / v$ and (b) $5 \% \mathrm{wt} / \mathrm{v}$. While large portions of the phase boundaries in neutral and basic conditions overlap, the phase boundaries in acidic conditions exhibit nontrivial deviations owing to the influence of hydrophobic interactions and hydrogen bonding on the phase behavior of the complexes.

parameter, $\chi_{e f f}$ as $\chi_{e f f}=\chi_{0}-w_{c} / \kappa^{2}$, where $w_{c}$ is the strength of the screened Coulombic interaction among monomers, and $\kappa^{2}$ is the inverse-square Debye screening length, proportional to salt concentration, and $\chi_{0}$ being the intrinsic interaction parameters. ${ }^{4,70}\left(\chi_{e f f}=0.5\right.$ has been reported for PAA in salt free aqueous solutions.71,72 Increasing salt concentration increases $\kappa^{2}$, leading to a $\chi_{e f f}>0.5$ signifying unfavorable interactions between PAA and water in saline solutions. Unsurprisingly, a quantitative prediction of the $\mathrm{pH}$ asymmetric salt resistance of PAA-PDMAEMA PECs was obtained by including a Flory-Huggins interaction parameter $\chi=0.75$ representing the interactions between PAA and water into the classic Voorn-Overbeek model for PEC phase behavior. ${ }^{36}$ 
PEC phase behavior in different $\mathrm{pH}$ conditions were collectively compared in Figure 6 showing the polymer-salt compositions for PECs prepared at (a) $C_{P, 0}=1 \% w t / v$ and (b) $C_{P, 0}=5 \% w t / v$. While the compositions of PECs starting with $C_{P, 0}=1 \% w t / v$ obtained at $\mathrm{pH}=3,6.5$, and 9 were similar, the trends with increasing $C_{S \text {, added }}$ were quantitatively divergent (Figure 6a). The divergence increased in the PECs starting with $C_{P, 0}=5 \%$ wt/v (Figure $6 \mathbf{b}$ ), which can be ascribed to stronger hydrophobicity and hydrogen bonding effects accompanying higher polymer concentrations. These effects also limited the accessible limits of salt additions, wherein the highest $C_{S, \text { added }}$ in $5 \% w t / v$ samples was $2.5 \mathrm{M}$ compared to 4.0 $\mathrm{M}$ for $1 \% w t / v$ samples.

In summary, the compositions of the PECs prepared in basic and neutral conditions were similar. The general trends, including screening effect of salt, self-suppression, and preferential partitioning of counterions, were also similar in the two cases. In neutral conditions, both polymers were fully ionized. In basic conditions, although PAH was partially ionized (PAA was completely ionized), the complexation behavior was not significantly affected. The association of ionized amino groups in PAH with carboxyl groups in PAA, facilitated by hydroxide ions in the aqueous solutions, is expected to result in the formation of stable complexes. However, PECs prepared in acidic conditions showed entirely different behavior. In these conditions, only a fraction of the PAA monomers were ionized and the polyelectrolyte chains precipitated in high salt conditions, rendering it unable to complex associatively with PAH. The solid precipitates that formed in low $\mathrm{pH}$ conditions comprised both pure PAA precipitates (with phase separation dominated by backbone hydrophobicity and hydrogen bonding) and PAA-PAH complexes, which led to higher PAA content in precipitates and an increase in the polymer content in the precipitates upon addition of salt. The unusual PAA accumulation in the phase-separated complexes in acidic conditions can therefore be surmised to occur with associative and segregative phase separation working simultaneously. In this sense, the phase behavior of PAA and PAH system, instead of being understood as simple polyelectrolyte complexation, can be regarded to emerge from a combined action of various intermolecular interaction, including electrostatics, hydrophobicity, and hydrogen bonding. The experimentally measured compositional maps reflect the superimposed result of these intermolecular interactions as a function of polymer concentration, salt concentration, and $\mathrm{pH}$.

Altogether, this investigation brought attention to the fact that the complexation of oppositely charged polyelectrolytes is a fairly complicated phenomena driven by not only electrostatic forces and entropic gains from counterion release, ${ }^{73,74}$ but also solvent effect and unavoidable intermolecular interactions. The nature of the phase separation process may change from electrostatic associative complexation to solvent-effect driven segregative precipitation. Oftentimes, such factors in realistic materials like PAA and PAH are overlooked or assumed to be inconsequential compared to charge-driven complexation mechanisms. To incorporate more of these advanced features into a wider materials infrastructure, we anticipate that theory and simulations can more easily interrogate the interplay of such variables. This experimental work elucidated molecular level insight of various complexation effects for the model polymer pairing of $\mathrm{PAA}(\mathrm{Na})$ and $\mathrm{PAH}$, which can enable greater comprehension and control over the structureproperty relationship of synthetic polymeric systems in micellar and gel-like architectures. ${ }^{75,76}$

\section{ASSOCIATED CONTENT}

\section{Supporting Information}

Materials and methods, supplementary figures and supplementary tables as Supporting Information are included. The Supporting Information is available free of charge on the ACS Publications website.

\section{AUTHOR INFORMATION}

\section{Corresponding Author}

*Email: samsri@ucla.edu

*E-mail: mtirrell@uchicago.edu

\section{Author Contributions}

S.S. and M.V.T. conceptualized the study. S.S. and L.L. designed the experiments. L.L. performed the experiments. L.L., S.S., J.M.T and M.V.T. contributed to the analysis/interpretation of the data and wrote the manuscript. S.S. and M.V.T. supervised the experimental work.

\section{Funding Sources}

This work was performed under the following financial assistance award 70NANB19H005 from U.S. Department of Commerce, National Institute of Standards and Technology (NIST) as part of the Center for Hierarchical Materials Design (CHiMaD) and MRSEC grant DMR-1420709 from U.S. National Science Foundation. J.M.T. acknowledges financial support from the NIST-CHiMaD Postdoctoral Fellowship.

\section{ACKNOWLEDGMENTS}

The authors acknowledge Dr. Kazi Sadman for helpful discussions. We also acknowledge the support from Dr. J. Juller from the James Franck Institute at the University of Chicago during thermogravimetric analysis.

\section{REFERENCES}

(1) Jong, B. de; Kruyt, H. G.; R, H. Koazervation. Proc. K. Ned. Akad. Wet. 1929, 32, 849-856.
Voorn, M. J. Complex Coacervation., Wiley-VCH Verlag GmbH Co. Amsterdam, 1956.

Overbeek, J. T. G.; Voorn, M. J. Phase Separation in Polyelectrolyte Solutions. Theory of Complex Coacervation. J. Cell. Comp. Physiol. 1957, 49, 7-26. Muthukumar, M. 50th Anniversary Perspective: A 
Perspective on Polyelectrolyte Solutions. Macromolecules 2017, 50, 9528-9560.

(5) Samanvaya, S.; Tirrell, M. V. Polyelectrolyte Complexation. Adv. Chem. Phys. 2016, 161, 499-544.

(6) Brangwynne, C. P.; Tompa, P.; Pappu, R. V. Polymer Physics of Intracellular Phase Transitions. Nat. Phys. 2015, 11, 899-904.

(7) Strom, A. R.; Emelyanov, A. V.; Mir, M.; Fyodorov, D. V.; Darzacq, X.; Karpen, G. H. Phase Separation Drives Heterochromatin Domain Formation. Nature 2017, 547, 241-245.

(8) Wang, Q.; Schlenoff, J. B. Single- and Multicompartment Hollow Polyelectrolyte Complex Microcapsules by One-Step Spraying. Adv. Mater. 2015, 27 (12), 2077-2082.

(9) Srivastava, S.; Andreev, M.; Levi, A. E.; Goldfeld, D. J.; Mao, J.; Heller, W. T.; Prabhu, V. M.; De Pablo, J. J.; Tirrell, M. V. Gel Phase Formation in Dilute Triblock Copolyelectrolyte Complexes. Nat. Commun. 2017, 8, 14131.

(10) Aumiller, W. M.; Pir Cakmak, F.; Davis, B. W.; Keating, C. D. RNA-Based Coacervates as a Model for Membraneless Organelles: Formation, Properties, and Interfacial Liposome Assembly. Nat. Chem. 2016, 32 (39), 10042-10053.

(11) Aumiller, W. M.; Keating, C. D. PhosphorylationMediated RNA/Peptide Complex Coacervation as a Model for Intracellular Liquid Organelles. Nat. Chem. 2016, 8 (2), 129-137.

(12) Meng, X.; Schiffman, J. D.; Perry, S. L. Electrospinning Cargo-Containing Polyelectrolyte Complex Fibers: Correlating Molecular Interactions to Complex Coacervate Phase Behavior and Fiber Formation. Macromolecules 2018, 51 (21), 8821-8832.

(13) Meng, X.; Perry, S. L.; Schiffman, J. D. Complex Coacervation: Chemically Stable Fibers Electrospun from Aqueous Polyelectrolyte Solutions. ACS Macro Lett. 2017, 6 (5), 505-511.

(14) Kuo, C. H.; Leon, L.; Chung, E. J.; Huang, R. T.; Sontag, T. J.; Reardon, C. A.; Getz, G. S.; Tirrell, M.; Fang, Y. Inhibition of Atherosclerosis-Promoting MicroRNAs via Targeted Polyelectrolyte Complex Micelles. J. Mater. Chem. B 2014, 2 (46), 8142-8153.

(15) Black, K. A.; Priftis, D.; Perry, S. L.; Yip, J.; Byun, W. Y.; Tirrell, M. Protein Encapsulation via Polypeptide Complex Coacervation. ACS Macro Lett. 2014, 3 (10), 1088-1091.

(16) Krogstad, D. V.; Lynd, N. A.; Miyajima, D.; Gopez, J.; Hawker, C. J.; Kramer, E. J.; Tirrell, M. V. Structural Evolution of Polyelectrolyte Complex Core Micelles and Ordered-Phase Bulk Materials. Macromolecules 2014, 47 (22), 8026-8032.

(17) Priftis, D.; Tirrell, M. Phase Behaviour and Complex Coacervation of Aqueous Polypeptide Solutions. Soft Matter 2012, 8 (36), 9396-9405.

(18) Fares, H. M.; Schlenoff, J. B. Diffusion of Sites versus
Polymers in Polyelectrolyte Complexes and Multilayers. J. Am. Chem. Soc. 2017, 139 (41), 1465614667.

Gucht, J. van der; Spruijt, E.; Lemmers, M.; Cohen Stuart, M. A. Polyelectrolyte Complexes: Bulk Phases and Colloidal Systems. J. Colloid Interface Sci. 2011, 361 (2), 407-422.

(20) Arthur, V.; Edward, B.; Shirley, M. Molecular Weight Fractionation and the Self-suppression of Complex Coacervation. Biopolymers 1967, 5 (1), 37-59.

(21) Salehi, A.; Desai, P. S.; Li, J.; Steele, C. A.; Larson, R. G. Relationship between Polyelectrolyte Bulk Complexation and Kinetics of Their Layer-by-Layer Assembly. Macromolecules 2015, 48 (2), 400-409.

(22) Bucur, C. B.; Sui, Z.; Schlenoff, J. B. Ideal Mixing in Polyelectrolyte Complexes and Multilayers: Entropy Driven Assembly. J. Am. Chem. Soc 2006, 128 (42), 13690-13691.

(23) Fu, J.; Schlenoff, J. B. Driving Forces for Oppositely Charged Polyion Association in Aqueous Solutions: Enthalpic, Entropic, but Not Electrostatic. J. Am. Chem. Soc. 2016, 138 (3), 980-990.

(24) Qin, J.; De Pablo, J. J. Criticality and Connectivity in Macromolecular Charge Complexation. Macromolecules 2016, 49 (22), 8789-8800.

(25) Kudlay, A.; De la Cruz, M. O. Precipitation of Oppositely Charged Polyelectrolytes in Salt Solutions. J. Chem. Phys. 2004, 120 (1), 404-412.

(26) Kudlay, A.; Ermoshkin, A. V.; De La Cruz, M. O. Complexation of Oppositely Charged Polyelectrolytes: Effect of Ion Pair Formation. Macromolecules 2004, 37 (24), 9231-9241.

(27) Perry, S. L.; Sing, C. E. PRISM-Based Theory of Complex Coacervation: Excluded Volume versus Chain Correlation. Macromolecules 2015, 48 (14), 50405053.

(28) Radhakrishna, M.; Basu, K.; Liu, Y.; Shamsi, R.; Perry, S. L.; Sing, C. E. Molecular Connectivity and Correlation Effects on Polymer Coacervation. Macromolecules 2017, 50 (7), 3030-3037.

(29) Chang, L. W.; Lytle, T. K.; Radhakrishna, M.; Madinya, J. J.; Vélez, J.; Sing, C. E.; Perry, S. L. Sequence and Entropy-Based Control of Complex Coacervates. Nat. Commun. 2017, 8, 1273.

(30) Zhang, P.; Alsaifi, N. M.; Wu, J.; Wang, Z. G. SaltingOut and Salting-In of Polyelectrolyte Solutions: A Liquid-State Theory Study. Macromolecules 2016, 49 (24), 9720-9730.

(31) Peng, B.; Muthukumar, M. Modeling Competitive Substitution in a Polyelectrolyte Complex. J. Chem. Phys. 2015, 143 (24), 243133.

(32) Priftis, D.; Laugel, N.; Tirrell, M. Thermodynamic Characterization of Polypeptide Complex Coacervation. Langmuir 2012, 28 (1), 15947-15957.

(33) Vitorazi, L.; Ould-Moussa, N.; Sekar, S.; Fresnais, J.; Loh, W.; Chapel, J. P.; Berret, J. F. Evidence of a Two- 
Step Process and Pathway Dependency in the Thermodynamics of Poly(Diallyldimethylammonium Chloride)/Poly(Sodium Acrylate) Complexation. Soft Matter 2014, 10 (47), 9496-9505.

(34) Wu, H.; Ting, J. M.; Werba, O.; Meng, S.; Tirrell, M. V. Non-Equilibrium Phenomena and Kinetic Pathways in Self-Assembled Polyelectrolyte Complexes. J. Chem. Phys. 2018, MACRO2018 (1), 163330.

(35) Takahashi, R.; Narayanan, T.; Sato, T. Growth Kinetics of Polyelectrolyte Complexes Formed from Oppositely-Charged Homopolymers Studied by Time-Resolved Ultra-Small-Angle X-Ray Scattering. J. Phys. Chem. Lett. 2017, 8 (4), 737-741.

(36) Jha, P. K.; Desai, P. S.; Li, J.; Larson, R. G. PH and Salt Effects on the Associative Phase Separation of Oppositely Charged Polyelectrolytes. Polymers (Basel). 2014, 6 (5), 1414-1436.

(37) Rumyantsev, A. M.; Zhulina, E. B.; Borisov, O. V. Complex Coacervate of Weakly Charged Polyelectrolytes: Diagram of States. Macromolecules 2018, 51 (10), 3788-3801.

(38) Sato, H.; Nakajima, A. Complex Coacervation in Sulfated Polyvinyl Alcohol- Aminoacetalyzed Polyvinyl Alcohol System - II. Formation of Coacervate Droplets. Colloid Polym. Sci. Kolloid Zeitschrift Zeitschrift für Polym. 1974, 252 (11), 944-948.

(39) Sato, H.; Nakajima, A. Complex Coacervation in Sulfated Polyvinyl Alcohol- Aminoacetalyzed Polyvinyl Alcohol System - I. Conditions for Complex Coacervation. Colloid Polym. Sci. Kolloid Zeitschrift Zeitschrift für Polym. 1974, 252 (11), 294-297.

(40) Friedowitz, S.; Salehi, A.; Larson, R. G.; Qin, J. Role of Electrostatic Correlations in Polyelectrolyte Charge Association. J. Chem. Phys. 2018, 149 (16), 163335.

(41) Salehi, A.; Larson, R. G. A Molecular Thermodynamic Model of Complexation in Mixtures of Oppositely Charged Polyelectrolytes with Explicit Account of Charge Association/Dissociation. Macromolecules 2016, 49 (24), 9706-9719.

(42) Sadman, K.; Wang, Q.; Chen, Y.; Keshavarz, B.; Jiang, Z.; Shull, K. R. Influence of Hydrophobicity on Polyelectrolyte Complexation. Macromolecules 2017, 50 (23), 9417-9426.

(43) Spruijt, E.; Westphal, A. H.; Borst, J. W.; Cohen Stuart, M. A.; Van Der Gucht, J. Binodal Compositions of Polyelectrolyte Complexes. Macromolecules 2010, 43 (15), 6476-6484.

(44) Chollakup, R.; Beck, J. B.; Dirnberger, K.; Tirrell, M.; Eisenbach, C. D. Polyelectrolyte Molecular Weight and Salt Effects on the Phase Behavior and Coacervation of Aqueous Solutions of Poly(Acrylic Acid) Sodium Salt and Poly(Allylamine) Hydrochloride Hydrochloride. Macromolecules 2013, 46 (6), 2376-2390.

(45) Boas, M.; Burman, M.; Yarin, A. L.; Zussman, E. Electrically-Responsive Deformation of
Polyelectrolyte Complex (PEC) Fibrous Membrane. Polymer (Guildf). 2018, 158, 262-269.

(46) Zhang, Y.; Li, F.; Valenzuela, L. D.; Sammalkorpi, M.; Lutkenhaus, J. L. Effect of Water on the Thermal Transition Observed in Poly(Allylamine Hydrochloride)-Poly(Acrylic Acid) Complexes. Macromolecules 2016, 49 (19), 7563-7570.

(47) Reisch, A.; Roger, E.; Phoeung, T.; Antheaume, C.; Orthlieb, C.; Boulmedais, F.; Lavalle, P.; Schlenoff, J. B.; Frisch, B.; Schaaf, P. On the Benefits of Rubbing Salt in the Cut: Self-Healing of Saloplastic PAA/PAH Compact Polyelectrolyte Complexes. Adv. Mater. 2014, 26 (16), 2547-2551.

(48) Zhao, M.; Xia, X.; Mao, J.; Wang, C.; Dawadi, M. B.; Modarelli, D. A.; Zacharia, N. S. Composition and Property Tunable Ternary Coacervate: Branched Polyethylenimine and a Binary Mixture of a Strong and Weak Polyelectrolyte. Mol. Syst. Des. Eng. 2019, 4, 110-121.

(49) Chollakup, R.; Smitthipong, W.; Eisenbach, C. D.; Tirrell, M. Phase Behavior and Coacervation of Aqueous Poly(Acrylic Acid)-Poly(Allylamine) Solutions. Macromolecules 2010, 43 (5), 2518-2528.

(50) Fu, J.; Fares, H. M.; Schlenoff, J. B. Ion-Pairing Strength in Polyelectrolyte Complexes. Macromolecules 2017, 50 (3), 1066-1074.

(51) Weidman, J. L.; Mulvenna, R. A.; Boudouris, B. W.; Phillip, W. A. Unusually Stable Hysteresis in the PHResponse of Poly(Acrylic Acid) Brushes Confined within Nanoporous Block Polymer Thin Films. J. Am. Chem. Soc. 2016, 138 (22), 7030-7039.

(52) Petrov, A. I.; Antipov, A. A.; Sukhorukov, G. B. BaseAcid Equilibria in Polyelectrolyte Systems: From Weak Polyelectrolytes to Interpolyelectrolyte Complexes and Multilayered Polyelectrolyte Shells. Macromolecules 2003, 36 (26), 10079-10086.

(53) Lappan, U.; Wiesner, B.; Scheler, U. Segmental Dynamics of Poly(Acrylic Acid) in Polyelectrolyte Complex Coacervates Studied by Spin-Label EPR Spectroscopy. Macromolecules 2016, 49 (22), 8616-8621. Huang, S.; Zhao, M.; Dawadi, M. B.; Cai, Y.; Lapitsky, Y.; Modarelli, D. A.; Zacharia, N. S. Effect of Small Molecules on the Phase Behavior and Coacervation of Aqueous Solutions of Poly(Diallyldimethylammonium Chloride) and Poly(Sodium 4-Styrene Sulfonate). J. Colloid Interface Sci. 2018, 518, 216-224.

(55) Aberkane, L.; Jasniewski, J.; Gaiani, C.; Scher, J.; Sanchez, C. Thermodynamic Characterization of Acacia Gum- $\beta$-Lactoglobulin Complex Coacervation. Langmuir 2010, 26 (15), 12523-12533.

(56) Zhao, M.; Zacharia, N. S. Sequestration of Methylene Blue into Polyelectrolyte Complex Coacervates. Macromol. Rapid Commun. 2016, 37 (15), 1249-1255.

Wang, S.; Chen, K.; Kayitmazer, A. B.; Li, L.; Guo, X. Tunable Adsorption of Bovine Serum Albumin by 
Annealed Cationic Spherical Polyelectrolyte Brushes. Colloids Surfaces B Biointerfaces 2013, 107, 251-256.

(58) Wang, Q.; Schlenoff, J. B. The Polyelectrolyte Complex/Coacervate Continuum. Macromolecules 2014, 47 (9), 3108-3116.

(59) Zhang, Y.; Yildirim, E.; Antila, H. S.; Valenzuela, L. D.; Sammalkorpi, M.; Lutkenhaus, J. L. The Influence of Ionic Strength and Mixing Ratio on the Colloidal Stability of PDAC / PSS Polyelectrolyte Complexes. Soft Matter 2015, 11 (37), 7392-7401.

(60) Perry, S. L.; Li, Y.; Priftis, D.; Leon, L.; Tirrell, M. The Effect of Salt on the Complex Coacervation of Vinyl Polyelectrolytes. Polymers (Basel). 2014, 6 (6), 17561772.

(61) Li, L.; Srivastava, S.; Andreev, M.; Marciel, A. B.; De Pablo, J. J.; Tirrell, M. V. Phase Behavior and Salt Partitioning in Polyelectrolyte Complex Coacervates. Macromolecules 2018, 51 (8), 2988-2995.

(62) Zhang, P.; Shen, K.; Alsaifi, N. M.; Wang, Z. G. Salt Partitioning in Complex Coacervation of Symmetric Polyelectrolytes. Macromolecules 2018, 51 (15), 55865593.

(63) Lytle, T. K.; Sing, C. E. Tuning Chain Interaction Entropy in Complex Coacervation Using Polymer Stiffness, Architecture, and Salt Valency. Mol. Syst. Des. Eng. 2018, 3 (1), 183-196.

(64) Mu, M.; Rieser, T.; Lunkwitz, K.; Meier-haack, J. Polyelectrolyte Complex Layers: A Promising Concept for Anti- Fouling Coatings Verified by inSitu ATR-FTIR Spectroscopy. Macromol. Rapid Commun. 1999, 20 (12), 607-611.

(65) Cranford, S. W.; Ortiz, C.; Buehler, M. J. Mechanomutable Properties of a PAA / PAH Polyelectrolyte Complex: Rate Dependence and Ionization Effects on Tunable Adhesion Strength. Soft Matter 2010, 6 (17), 4175-4188.

(66) Rathee, V. S.; Zervoudakis, A. J.; Sidky, H.; Sikora, B. J.; Whitmer, J. K. Weak Polyelectrolyte Complexation Driven by Associative Charging. J. Phys. Chem. 2018, 148 (11), 114901.
Rathee, V. S.; Sidky, H.; Sikora, B. J.; Whitmer, J. K.
Role of Associative Charging in the Entropy-Energy Balance of Polyelectrolyte Complexes. J. Am. Chem. Soc 2018, 140, 15319-15328.

(68) Flory, P. J.; Osterheld, J. E. Intrinsic Viscosities of Polyelectrolytes. Poly-(Acrylic Acid). J. Phys. Chem. 1954, 58 (8), 653-661.

(69) Buscall, R.; Corner, T. The Phase Separation Behaviour of Aqueous Solutions of Polyacrylic Acid and Its Partial Sodium Salts in the Presence of Sodium Chloride. Eur. Polym. J. 1982, 18, 967-974.

(70) Prabhu, V. M.; Muthukumar, M.; Wignall, G. D.; Melnichenko, Y. B. Polyelectrolyte Chain Dimensions and Concentration Fluctuations near Phase Boundaries. J. Chem. Phys. 2003, 119 (7), 4085-4098.

(71) Thakur, A.; Wanchoo, R. K.; Singh, P. Structural Parameters and Swelling Behavior of PH Sensitive Poly(Acrylamide-Co-Acrylic Acid) Hydrogels. Chem. Biochem. Eng. Q. 2011, 25 (2), 181-194.

(72) Eustace, D. J.; Siano, D. B.; Drake, E. N. Polymer Compatibility and Interpolymer Association in the Poly(Acrylic Acid)-Polyacrylamide-Water Ternary System. J. Appl. Polym. Sci. 1988, 35 (3), 707-716.

(73) Borue, V. Y.; Erukhimovich, I. Y. A Statistical Theory of Weakly Charged Polyelectrolytes: Fluctuations, Equation of State and Microphase Separation. Macromolecules 1988, 21 (11), 3240-3249.

(74) Ou, Z.; Muthukumar, M. Entropy and Enthalpy of Polyelectrolyte Complexation: Langevin Dynamics Simulations. J. Chem. Phys. 2006, 124 (15), 154902.

(75) Ting, J. M.; Wu, H.; Herzog-Arbeitman, A.; Srivastava, S.; Tirrell, M. V. Synthesis and Assembly of Designer Styrenic Diblock Polyelectrolytes. ACS Macro Lett. 2018, 7 (6), 726-733.

(76) Hunt, J. N.; Feldman, K. E.; Lynd, N. A.; Deek, J.; Campos, L. M.; Spruell, J. M.; Hernandez, B. M.; Kramer, E. J.; Hawker, C. J. Tunable, High Modulus Hydrogels Driven by Ionic Coacervation. Adv. Mater. 2011, 23 (20), 2327-2331. 
Table of Contents Figure

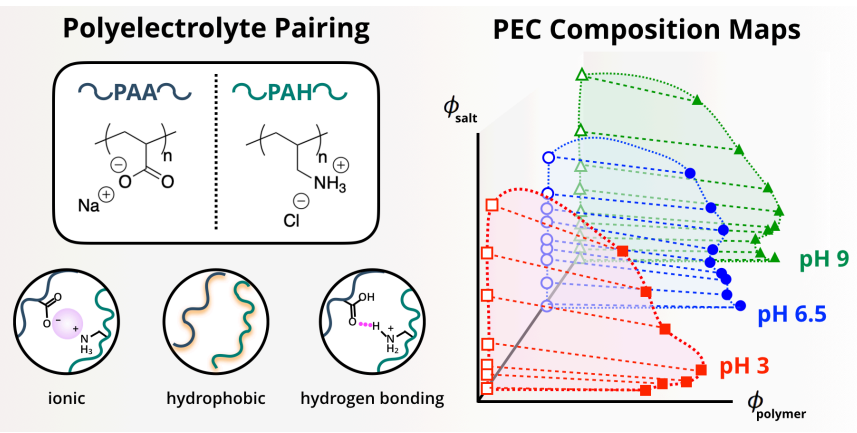

